\title{
Deep Learning Based Techniques for Sentiment Analysis: A Survey
}

Wael Etaiwi

King Talal School of Business Technology, Princess Sumaya University for Technology, Amman, Jordan

E-mail: w.etaiwi@psut.edu.jo1

Dima Suleiman

King Abdullah II School of Information Technology, The University of Jordan, Amman, Jordan

E-mail: dima.suleiman@ju.edu.jo2

Arafat Awajan

King Hussein School of Computing Sciences, Princess Sumaya University for Technology, Amman, Jordan

College of Information Technology, Mutah University, AlKarak

E-mail: Jordan, awajan@mutah.edu.jo4

\section{Overview Paper}

Keywords: natural nanguage processing, sentiment analysis, deep learning

Received: August 8, 2021

\begin{abstract}
The automated representation of human language using a variety of techniques is called Natural Language Processing (NLP). Improvements to NLP applications are important and can be accomplished using a variety of methods, such as graphs, deep neural networks, and word embedding. Sentiment classification, which attempts to automatically classify opinionated text as positive, negative, or neutral, is a fundamental activity of sentiment analysis. Sentiment analysis methods focused on deep learning over the past five years are analyzed in this review.
\end{abstract}

Povzetek: Opisan je kratek pregled metod globokega učenja za določanje razpoloženja iz besedil.

\section{Introduction}

Sentiment analysis is an NLP application that extracts and analyzes the subjective information of the writer attitudes and opinions in various domains such as politics, sociology, business intelligence, etc. [1]. Moreover, the sentiment classification within certain domain could be about any event, item, topic, product, mobile application, or others. Sentiment analysis is important in many different tasks such as digital mental health [2] and recommender systems [3]. Furthermore, Sentiment analysis is also used in actual intelligent technologies for mental health, like intelligent cognitive assistants [4]. The main aim of sentiment analysis is to classify text into what is called polarity [5]. There are several forms of polarity, including the sentiment class, which can have several values, such as very positive, positive, neutral, negative and very negative, where the polarity values and classes differ from one study to another. Sentiment expressions such as happy, sad, angry, etc., in addition to sentiment classes, may be analyzed in sentiment analysis. In addition, text classification can be subjective or objective [6].

Sentiment analysis approaches are classified intro three main groups [5]: Lexicon-based approaches, machine learning approaches, and hybrid approaches. In lexicon-based approaches, the polarity is extracted based on a predefined lexicon or dictionary. A sentiment dictionary refers to a list of words and their corresponding polarity value. While corpus is a dataset used to train the sentiment analysis model. On the other hand, in machine learning approaches, the computer is trained over a given dataset in order to build a classification model that could be used to extract the sentiment polarity of a given text. Finally, the hybrid approach combines both lexicon-based and machine learning technique to perform the sentiment analysis task.

One of the main machine learning techniques is called a Neural Network (NN). NN is a programming paradigm that enables a computer to learn from observed data. NN is a biologically inspired structure that consists of a large number of interconnected processing units, called neurons, working to solve a specific problem or to enhance a specific application, such as image processing or pattern recognition. It consists of three main levels or layers (see Figure 1): the input layer, the hidden layer, and the output layer [7]. Neurons in the input layer (input neurons) are activated through sensing the environment, and then they produce activation values (weights) passed to neurons in the next layer and activate them. 


\section{Hidden Layer}

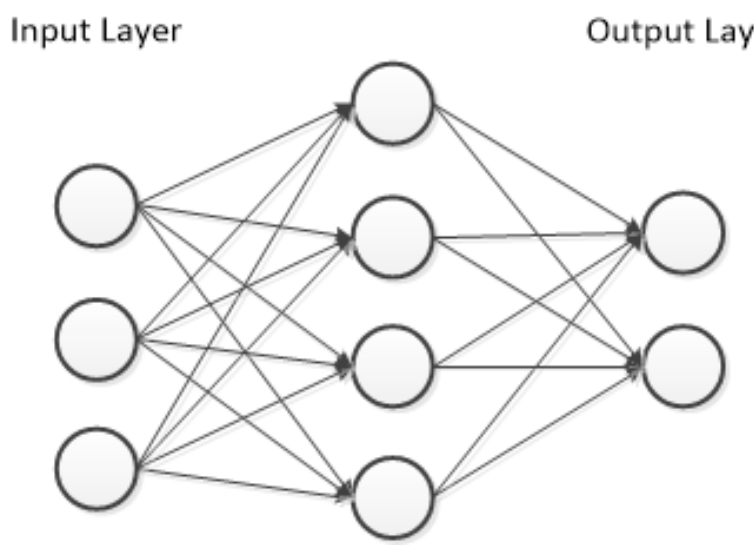

Figure 1: Basic structure of neural network.

Deep learning is a sub-field of machine learning that exploits many levels of representation (layers) that contain information processing units (neurons) for feature extraction and transformation, and pattern analysis and classification [8]. Deep learning provides better data representation that can make it easier to learn using machine learning techniques.

Deep learning enhances different computer tasks, including text mining, image processing, and pattern recognition. By using deep learning, it is possible to represent words from textual data and produce word embedding that could be used by different machine learning techniques.

Deep learning is used to learn features, rather than handcraft them. Handcrafting features is a timeconsuming process and is often incomplete. However, deep learning provides good features and multiple levels of representation that improve the efficiency of machine learning models.

Several types of deep neural networks are used for NLP such as feedforward NN, Convolutional Neural Network (CNN), and Recurrent Neural Network (RNN). Deep learning outperforms other machine learning methods in several NLP tasks such as machine translation and named-entity recognition [9], [10]. Hence, several deep learning methods have been introduced to solve various NLP tasks. Yet supervised learning is the most widely used solution to NLP in recent deep learning studies [9].

The purpose of this paper is to provide a comprehensive survey of the sentiment analysis based on deep learning. The recent proposed deep learning-based approaches for sentiment analysis are further classified according to the type of deep learning network used. Three different categories are used: feed forward neural network-based sentiment analysis approaches, CNN based sentiment analysis approaches, and RNN based sentiment analysis approaches. Most of the proposed sentiment analysis surveys throughout the previous few decades have been focused on the primary methodologies used to identify text polarity. Many surveys categorize the proposed sentiment analysis approaches as machine learning-based techniques, lexicon-based techniques, or hybrid strategies [5]. Because Deep Learning is growing and producing promising results in a variety of applications, the primary focus of this review is on Deep Learning-based techniques used for sentiment analysis. The remainder of this paper is structured as follows: section 2 discusses the methodology used to perform this review research; section 3 includes a discussion of the research analyzed for sentiment analysis using deep learning. In section 4, the analysis and conclusion are discussed.

\section{Methodology}

The researches available on the ScienceDirect ${ }^{1}$, the ACM digital library ${ }^{2}$ and IEEE Xplore Digital library ${ }^{3}$ were used as data sources in this review. Specific keywords and their synonyms are used to select the articles to be includes in this review. The keywords selected are used to select the most suitable articles that fit the review topic. Deep learning-based sentiment, deep neural network sentiment, or multilayer neural network sentiment is a search string used in the paper search method.

The search string is adjusted to significantly reduce the number of studies that are irrelevant. The search is limited for the last five years of publication. Journaled papers and conference or proceedings are among the publications included. The number of paper articles included is limited to those that target English text.

When the paper article search stage is completed, a large number of papers that meet the criteria will be filtered out during the search adjustment process. The inclusion and exclusion criteria were established, and they were used to decide which papers to include or exclude from this review. Articles that use deep leaning neural network for sentiment analysis and explicitly discuss the key methodology, the dataset, the deep learning network type and structure, and the experimental evaluation meet the inclusion requirements. Journal and conference paper researches are included in this collection of articles. The chosen articles were published between 2015 and 2020 . Articles that lack experimental evidence, a consistent dataset, a clear deep neural network architecture, and a clear methodology are among the exclusion criteria. Articles that focused at topics other than sentiment analysis and targeted languages other than English were also excluded.

\section{Literature review}

A range of research related to the study of sentiment using deep learning techniques is briefly discussed in this section. Sentiment analysis tasks can be carried out effectively by applying various models, such as deep learning models, which have recently been expanded.

\footnotetext{
${ }^{1}$ https://www.sciencedirect.com/

2 https://dl.acm.org/
}

${ }^{3}$ https://ieeexplore.ieee.org/ 
These models cover all various types of deep learning networks including: Feed Forward Neural Network (FFNN), Convolutional Neural Network (CNN), and Recurrent Neural Network (RNN). This section describes the efforts to integrate deep learning models for sentiment analysis by various researchers.

\section{Feed Forward Neural Network (FFNN)}

Feedforward NN is the simplest form of deep neural networks. It has a front propagated wave using a classifying activation function. In feedforward NN, there are no feedback connections in which the outputs of the model are fed back into itself. The information only travels forward in the neural network, from the input layer then through the hidden layers and finally through the output layer. Thus, all the data is inputted once in the input layer, and no data will be fed through. Therefore, for sentence manipulation, an input node is required for each word in the sentence.

In their research [11], they studied the sentiment analysis of Arabic tweets because of the lack of applications that studies the Arabic sentiment since the Arabic language has different features. In their research, they proposed to use a hybrid approach. The first approach is the preprocessing which includes: stemming, tokenization, and stop word removal in addition to using two algorithms for weighting the features which are chai square and information gain. On the other hand, the second approach is the use of feed forward deep learning approach in Arabic tweets sentiment classification which used two polarities to classify the tweet into either positive or negative tweets. Moreover, comparisons were conducted using Arabic tweets and several classification methods such that: neural network, decision tree and support vector machine. As a conclusion, the hybrid approach achieved the highest results in terms of accuracy, precision with values $90 \%$ and $93.7 \%$ respectively.

\section{Convolutional Neural Network (CNN)}

A Convolutional Neural Network $(\mathrm{CNN})$ is a neural-based approach that represents a feature function that extracts higher-level features [9]. Coevolutionary filters are applied to all possible window sizes in $\mathrm{CNN}$ in the input layer to produce a function map. After that, a max-pooling operation is applied on each filter to obtain a fixed-length output in order to reduce the output dimensionality. For word-level applications (such as word-based prediction), a window-based approach is used in $\mathrm{CNN}$ where the fixedsize window of adjacent words is taken into account for each word in the input text. Then, in the center of the window, a standalone $\mathrm{CNN}$ is applied to the window to predict the term. In contextual windows, $\mathrm{CNN}$ is efficient in handling semantic clues, but fails to preserve sequential order and model long-distance contextual knowledge that is essential for different NLP tasks.

The main difference between the ordinary neural network or FFNN and CNN is the number of layers [7]. CNN contains several numbers of convolutional layers with nonlinear activation functions. The ordinary neural network has fully connected layers in which each input neuron is connected to each output neuron in the next layer. However, in $\mathrm{CNN}$, the output is computed using convolutions over the input layer. Therefore, each layer applies different filters (functions that analyze different features in the data [12]) and combine their results.

$\mathrm{CNN}$ has been used in many sentiment analyses, however, in their work [13], they tried to use CNN filters with several lengths and grasp their power to find the polarity of the sentence in order to enhance the performance of the CNN classifier. Moreover, they used the Adaboost to combine several classifiers based on the size of the filter where the Adaboost can learn the classification error of weak classifier, thus the weak classifier will be combined with strong one in order to improve the final classification by adjusting the weights. Also, the experiments were made on Movie Review and IMDB using several N-grams.

Convolutional neural networks (CNN) was not only used in text sentiment but it also was used in multimedia sentiments analysis which consists of text and images [14]. However, the input for $\mathrm{CNN}$ will be the combination of two individual CNN architectures, where one of them was used to learn the visual features and the other one was used to learn the text features. Thus, this helps in finding the internal relationship between the features. The experiments were made in two datasets: text sentiment (SentiStrength), visual sentiment (SentiBank) and the combination of the two, where the combined classifier of the two datasets provide the best results in predicting the sentiment. Moreover, the word embedding which was used to represent the text and images was skip-gram model and training was made in English Wikipedia corpus. In addition, comparisons were made between the proposed approach and several machine learning algorithms such as: SVM, Naïve Bays, and others. As a conclusion, the proposed CNN outperforms all other methods in term of accuracy with $79 \%$, especially when used over the combined dataset.

Different configuration settings of CNN were used by Rani and Kumar [15] in order to build a sentiment analysis model for Hindi movie reviews. The proposed model was trained over the $50 \%$ of the manually annotated dataset. The dataset was annotated by three native speakers of Hindi language. The experimental results were compared with the results of other machine learning approaches, and the comparison showed that the proposed CNN model overcome the performance of other state-of-the-art model and achieved an accuracy of $95 \%$.

Kapociute-Dzikiene et al. [16] applied CNN and LSTM deep learning neural networks to perform sentiment analysis on the Lithuanian Internet comment dataset. The proposed models were used on the top of both word2vec and FastText word embedding. The best result was reported using CNN with $70.6 \%$ of accuracy. The authors concluded that the deep learning neural networks demonstrated good results when applied to smaller dataset.

A hybrid deep learning model for fine-grained prediction of sentiment in real-time multimodal data was proposed by Kumar et al. [14]. To deal with two unique semiotic systems, the authors combined deep learning 
with machine learning: the textual (written text) and visual (still images) and their combination within the online content. There are four modules in the proposed model: discretization, text analysis, image analytics, and the decision module. Google Lens was used by the Discretization Module to separate text from images, which are then processed as separate entities and sent to the respective text analysis and image analysis modules. The Text Analytics Module explains the sentiment of using a CNN hybrid enriched with SentiCircle's contextual semantics. The $91 \%$ accuracy is obtained by the proposed model.

\section{Recurrent Neural Network (RNN)}

Recurrent Neural Network (RNN) is a neural-based approach that is effective in the processing of sequential information [9]. For each instance of the input sequence, RNN recursively uses the results previously computed. After being represented by a fixed-size token vector, the input sequences are fed sequentially (one by one) to the recurrent unit. The primary strength of an RNN is the ability to reuse previous computational results in future computations. Unlike FFNN, RNN makes it possible to feed input words through or feed input sequentially. RNN, on the other hand, continues to suffer from the issue of the vanishing gradient (changing previous results induces very minor changes in future computations), making it difficult to learn and modify the parameters in the earlier layers. [17].

The order of input data elements is a key factor in several applications [18]. For example, the word's order in a sentence can play a key role in NLP applications. Therefore, the need for some kind of memory to reserve the input data element order emerges. This is RNN's primary feature. The previous and the following terms may be important in NLP applications. Thus, when processing a word in such applications using two RNN layers and combining their outputs, it is important to look in both directions, forward and backwards. This mechanism is known as bidirectional RNN [19]. There are several different RNN types, such as Long Short-Term Memory (LSTM) [20] and Gated Recurrent Unit (GRU) [21]. In LSTM, for a short time during the learning process, the neurons are designed to retain, forget, or expose knowledge. Significant data can be preserved for longer by using LSTM, while irrelevant information can be forgotten. In GRU, relevant information is also retained using two main operations: reset and update, instead of three operations in LSTM. Hence, GRU performs as well as or better than LSTM.

The bidirectional and unidirectional nature of three types of RNN was considered in [22], where the three types are: GRU, LSTM and vanilla RNNs. Moreover, the performance evaluation of these three types was made using benchmark datasets SST-1 and SST-2 for sentiment analysis and health product review from Amazon, while the pre-training for word vector was made on Google News dataset. Accordingly, in term of accuracy, the GRU was the best. However, in case of the simple dataset and short reviews it is not recommended to use RNNs.
A new neural network model was proposed in [23] which is called Attention-based LSTM model with the Aspect information (ALA). However, the new model was proposed to predict the sentiment score of financial opinion mining and to assign it to a certain pre-defined class. Therefore, there are layers of LSTM that take the word embedding as input and keep the intermediate output to relate them to the representation of final distribution. In addition, the dataset was used in the experiment contains 675 microblog messages and 504 news headlines. Also, the sentiment score range between -1 and 1 and the number of classification labels were 27. As an input to the neural network, the word2vec model was used for word embedding. Moreover, the proposed model considered the aspect in order to precisely determine the word context. Accordingly, comparisons were made between several deep learning models and as a result, ALA got the best performance.

In their research [24], they predict the sentiment and aspect of financial text using different deep learning models in their ensemble approaches which are: Recurrent Neural Networks (RNNs) and Convolutional Neural Networks (CNNs). Moreover, for prediction, they used voting and ridge regression at the last step, while they used the word embedding to convert the input and trained it using Twitter corpus. As a result, the performance of using CNNs was better than using RNNs on both sentiment and Aspect prediction.

Chen et al. [25] proposed a sentiment analysis framework using a deep neural LSTM network in order to propose a self-developed military sentiment dictionary. The Militarylife PTT board of the largest online forum in Taiwan was used as the basis of the used dataset. The experimental results showed that the accuracy and F1measure of the proposed framework were higher than the results from using only existing dictionaries of sentiment.

LSTM was used by Ghulam et al. [26] to build a sentiment analysis model for Roman Urdu language. The experimental results showed the significant accuracy with compare to other machine learning methods.

Instead of having sentiment analysis at word or phrase level, in their research [27], they proposed a framework for sentiment analysis in document level. However, the input will be the document and the result will be an opinion or sentiment such as thumbs down and thumbs up. Therefore, in order to achieve document-level sentiment analysis, the document semantic representation is very crucial. Thus, they fragmented the semantics of the documents into four constitutes which are: representation of the word, the structure of the sentence, the composition of the sentence and the composition of the documents. The reason for this fragmentation is that, the meaning of the words and the rules that combine the words determine the semantics of the sentences and documents. On the other hand, the word embedding is used to represent the words to capture its meaning, but instead of using the standard word embedding, they used to learn sentiment specific word embedding (SSWE). The reason of using (SSWE) is to handle the problem of having sentiment words that are opposite in sentiment polarity but they are neighbors such as "good" and "bad" since they have similar context. 


\begin{tabular}{|c|c|c|c|c|}
\hline Paper & Year & $\begin{array}{l}\text { Neural Network } \\
\text { Model }\end{array}$ & Dataset & Results/Evaluation \\
\hline$[11]$ & 2017 & $\mathrm{FF}$ & Arabic tweet & $\begin{array}{l}\text { Accuracy, precision with } 90 \% \\
\text { and } 93.7 \% \text { respectively. }\end{array}$ \\
\hline [13] & 2016 & $\mathrm{CNN}$ & Movie Review and IMDB & \\
\hline$[14]$ & 2015 & $\mathrm{CNN}$ & $\begin{array}{l}\text { SentiStrength (text) } \\
\text { SentiBank (visual) }\end{array}$ & Accuracy $79 \%$ \\
\hline$[15]$ & 2018 & $\mathrm{CNN}$ & Manually annotated dataset & Accuracy $95 \%$ \\
\hline [30] & 2020 & $\mathrm{CNN}$ & 8000 comments and posts & Accuracy $90.9 \%$ \\
\hline$[16]$ & 2019 & $\begin{array}{l}\text { CNN and RNN- } \\
\text { LSTM }\end{array}$ & $\begin{array}{c}\text { Lithuanian Internet } \\
\text { comments }\end{array}$ & Accuracy $70.6 \%$ \\
\hline [22] & 2017 & RNN & $\begin{array}{l}\text { Amazon health product } \\
\text { reviews, SST-1 and SST-2 }\end{array}$ & $\begin{array}{c}\text { In term of accuracy, GRU is the } \\
\text { best }\end{array}$ \\
\hline [23] & 2018 & RNN-LSTM & $\begin{array}{l}504 \text { news headlines and } \\
675 \text { microblog messages }\end{array}$ & \\
\hline [24] & 2018 & RNN & $\begin{array}{c}\text { Twitter posts and news } \\
\text { headlines in the financial } \\
\text { domain. }\end{array}$ & \\
\hline [25] & 2019 & RNN-LSTM & Militarylife PTT & $\begin{array}{c}\text { Accuracy } 85.4 \% \\
\text { F1-measure } 88.41 \%\end{array}$ \\
\hline$[26]$ & 2019 & RNN-LSTM & Roman Urdu dataset & Accuracy $95.2 \%$ \\
\hline [27] & 2015 & Word embedding & SemEval 2013 & \\
\hline [28] & 2020 & Word embedding & Tweets & Accuracy $62.8 \%$ \\
\hline [29] & 2015 & Auto-encoder & $\begin{array}{c}\text { Linguistic Data Consortium } \\
\text { Arabic Tree Bank }\end{array}$ & Accuracy $73.5 \%$ \\
\hline
\end{tabular}

Table 1: Recently proposed sentiment analysis approaches.

According to the structure of the sentence, the sentence representation is affected by the representation of its words. Finally, the sentence representation and the document composition are used to represent the semantics of the document.

Sentiment Analysis of COVID-19 tweets were studied by Chakraborty et al. [28]. This study analyzed two types of tweets gathered during the pandemic times. Deep learning was used to validate the author's thoughts that people have tweeted positive regards COVID-19. In this work, two sets of datasets were used. Although one dataset contains all the tweets that were released during the timeframe from December 2019 to May 2020, the second collection of tweets that were most retweeted were given priority.

Arabic sentiment classification for sentence level was the main focus in [29]. In their work, Al Sallab and his colleagues proposed a deep learning framework which explored four several architectures. The first three architectures based on Bag-of-Words as an input along with features extracted from the Arabic sentiment lexicon. Thus, the three models were based on Deep AutoEncoders (DAE) and Deep Belief Networks (DBN). On the other hand, the fourth architecture was used to handle the shortage of context handling in previous three architectures which based on Recursive Auto Encoder (RAE). As a result, the fourth architecture was the best and had many advantages in term of no need to have a lexicon as a resource.
Deep Neural Network (DNN) used several layers of conventional neural network and applied back propagation, while DBN used the fine-tuning step but before using it, it applied pre-training. On the other hand, DAE reduces the dimensionality of the original model representation. However, RAE minimized the input data, probability by parsing the input words in the best order by exploring the best parse tree. Therefore, the result is the reorder of the input sentences in order to compact its representation. Also, this is the same result that was provided using DAE where the objective of both models was to minimize the reconstruction error. On the other side, the difference between the two models was that DAE can deal only with a vector that is fixed length, while RAE can parse sentences with variable length. However, both models based on unsupervised learning, and both of them require classifier at the top which was $\mathrm{DBN}$ in case of DAE and Softmax in case of the RAE. The experimental results showed that the F1 measure score was improved by $9 \%$ over all other literature results.

\section{Discussion and conclusion}

Deep learning is made up of a number of popular and effective models that are used to tackle a wide range of issues. In this review, various studies are reviewed in order to provide a thorough understanding of the successful growth of deep learning applications in the field of sentiment analysis. It is obvious from this survey that the work on applying deep neural networks for sentiment 
analysis is receiving growing attention in recent years. Table 1 summarizes recent papers on the various types of deep learning neural networks to construct sentiment analysis models in the last five years. The third column in Table 1 indicates that, various types of deep-learning neural networks could be used to achieve good results in sentiment analysis. The fourth column shows that there are several different datasets can be used to evaluate the proposed models. The lack of a single or benchmark dataset for this purpose makes it a difficult task to compare the different models together. A number of datasets is available for sentiment analysis, but the datasets used for deep learning are vary. On the other hand, the accuracy achieved by the listed researches are slightly different; the preferences for one module over another depends on the size of the dataset and the parameters of deep neural network. After reviewing all of these studies, it has been shown that sentiment analysis can be done more efficiently and accurately utilizing deep learning approaches. Deep learning networks perform autonomous feature extraction without the need for human interaction, which saves time by eliminating the requirement for feature engineering. Deep learning, on the other hand, necessitates a considerable amount of data as compared to other machine learning algorithms, which becomes its primary limitation. Word embeddings clearly played an important part in deep learning-based sentiment analysis algorithms. It is also clear that word embeddings can be used as features in non-neural learning models for a range of tasks that don not even require deep learning. This review presents a comprehensive survey of using deep learning for sentiment analysis. The studies are analyzed and classified according to the type of deep neural network used in the sentiment analysis models.

\section{References}

[1] G. Beigi, X. Hu, R. Maciejewski, and H. Liu, "An Overview of Sentiment Analysis in Social Media and Its Applications in Disaster Relief," in Sentiment Analysis and Ontology Engineering, Springer, Cham, 2016, pp. 313-340. Accessed: Jun. 02, 2018. https://doi.org/10.1007/978-3-319-30319-2_13

[2] T. Kolenik and M. Gams, "Intelligent Cognitive Assistants for Attitude and Behavior Change Support in Mental Health: State-of-the-Art Technical Review," Electronics, vol. 10, no. 11, p. 1250, Jan. 2021

https://doi.org/10.3390/electronics10111250.

[3] B. Alhijawi and A. Awajan, "Prediction of Movie Success using Twitter Temporal Mining," in Proceedings of the Sixth International Congress on Information and Communication Technology: ICICT 2021, London, vol. 1. https://doi.org/10.1007/978-981-16-2377-6_12

[4] T. Kolenik and M. Gams, "Persuasive Technology for Mental Health: One Step Closer to (Mental Health Care) Equality?," IEEE Technol. Soc. Mag., vol. 40, no. 1, pp. 80-86, Mar. 2021. https://doi.org/10.1109/MTS.2021.3056288.
[5] M. Biltawi, W. Etaiwi, S. Tedmori, A. Hudaib, and A. Awajan, "Sentiment classification techniques for Arabic language: A survey," 2016 7th Int. Conf. Inf. Commun. Syst. ICICS 2016, pp. 339-346, 2016. https://doi.org/10.1109/IACS.2016.7476075

[6] V. Rajput and S. Dubey, "An Overview of Use of Natural Language Processing in Sentiment Analysis based on User Opinions," Int. J. Adv. Res. Comput. Sci. Softw. Eng., vol. 6, no. 4, 2016.

[7] M. M. Lopez and J. Kalita, "Deep Learning applied to NLP," CoRR, vol. abs/1703.03091, 2017.

[8] L. Deng, "Deep Learning: Methods and Applications," Found. Trends® Signal Process., vol. 7, no. 3-4, pp. 197-387, 2014, https://doi.org/10.1561/2000000039.

[9] T. Young, D. Hazarika, S. Poria, and E. Cambria, "Recent trends in deep learning based natural language processing," Ieee Comput. Intell. Mag., vol. 13, no. 3, pp. 55-75, 2018. https://doi.org/10.0.4.85/MCI.2018.2840738

[10] L. Deng and Y. Liu, Eds., Deep Learning in Natural Language Processing. Springer Singapore, 2018. https://doi.org/10.1007/978-981-10-5209-5

[11] A. Altaher, "Hybrid approach for sentiment analysis of Arabic tweets based on deep learning model and features weighting," Int. J. Adv. Appl. Sci., vol. 4, no. 8, pp. 43-49, Aug. 2017. https://doi.org/10.21833/ijaas.2017.08.007.

[12] Y. LeCun, Y. Bengio, and others, "Convolutional networks for images, speech, and time series," Handb. Brain Theory Neural Netw., vol. 3361, no. 10, p. $1995,1995$. https://doi.org/10.5555/303568.303704

[13] Y. Gao, W. Rong, Y. Shen, and Z. Xiong, "Convolutional Neural Network based sentiment analysis using Adaboost combination," in 2016 International Joint Conference on Neural Networks (IJCNN), Jul. 2016, pp. 1333-1338. https://doi.org/10.1109/IJCNN.2016.7727352.

[14] G. Cai and B. Xia, "Convolutional Neural Networks for Multimedia Sentiment Analysis," in Natural Language Processing and Chinese Computing, Springer, Cham, 2015, pp. 159-167. Accessed: Jun. $01,2018$. https://doi.org/10.1007/978-3-319-25207-0_14

[15] S. Rani and P. Kumar, "Deep Learning Based Sentiment Analysis Using Convolution Neural Network," Arab. J. Sci. Eng., vol. 44, no. 4, pp. 3305-3314, Aug. 2018, https://doi.org/10.1007/s13369-018-3500-z.

[16] J. Kapociute-Dzikiene, R. Damaševičius, and M. Woźniak, "Sentiment Analysis of Lithuanian Texts Using Traditional and Deep Learning Approaches," Comput., vol. 8, p. 4, 2019. https://doi.org/10.3390/computers8010004

[17] P. Le and W. Zuidema, "Quantifying the Vanishing Gradient and Long Distance Dependency Problem in Recursive Neural Networks and Recursive LSTMs," in Proceedings of the 1st Workshop on Representation Learning for NLP, Berlin, Germany, 
Aug. 2016, pp. 87-93. https://doi.org/10.18653/v1/W16-1610.

[18] J. Schmidhuber, "Deep learning in neural networks: An overview," Neural Netw., vol. 61, pp. 85-117, Jan. 2015. https://doi.org/10.1016/j.neunet.2014.09.003.

[19] M. Schuster and K. K. Paliwal, "Bidirectional Recurrent Neural Networks," Trans Sig Proc, vol. 45, no. 11, pp. 2673-2681, Nov. 1997. https://doi.org/10.1109/78.650093.

[20] S. Hochreiter and J. Schmidhuber, "Long short-term memory," Neural Comput., vol. 9, no. 8, pp. 17351780, 1997. https://doi.org/10.1162/neco.1997.9.8.1735

[21] K. Cho, B. van Merriënboer, D. Bahdanau, and Y. Bengio, "On the Properties of Neural Machine Translation: Encoder-Decoder Approaches," in Proceedings of SSST-8, Eighth Workshop on Syntax, Semantics and Structure in Statistical Translation, Doha, Qatar, Oct. 2014, pp. 103-111. https://doi.org/10.3115/v1/W14-4012.

[22] K. Baktha and B. K. Tripathy, "Investigation of recurrent neural networks in the field of sentiment analysis," in 2017 International Conference on Communication and Signal Processing (ICCSP), Apr. 2017, pp. 2047-2050. https://doi.org/10.1109/ICCSP.2017.8286763.

[23] S. E., L. Yang, M. Zhang, and Y. Xiang, “Aspectbased Financial Sentiment Analysis with Deep Neural Networks," in Companion Proceedings of the The Web Conference 2018, Republic and Canton of Geneva, Switzerland, 2018, pp. 1951-1954. https://doi.org/10.1145/3184558.3191825.

[24] G. Piao and J. G. Breslin, "Financial Aspect and Sentiment Predictions with Deep Neural Networks: An Ensemble Approach," in Companion of the The Web Conference 2018 on The Web Conference
2018, 2018, pp. 1973-1977. https://doi.org/10.1145/3184558.3191829

[25] L.-C. Chen, C.-M. Lee, and M.-Y. Chen, "Exploration of social media for sentiment analysis using deep learning," Soft Comput., vol. 24, no. 11, pp. 8187-8197, Oct. 2019. https://doi.org/10.1007/s00500-019-04402-8.

[26] H. Ghulam, F. Zeng, W. Li, and Y. Xiao, "Deep Learning-Based Sentiment Analysis for Roman Urdu Text," Procedia Comput. Sci., vol. 147, pp. 131-135, 2019. https://doi.org/10.1016/j.procs.2019.01.202.

[27] D. Tang, "Sentiment-Specific Representation Learning for Document-Level Sentiment Analysis,' in Proceedings of the Eighth ACM International Conference on Web Search and Data Mining, New York, NY, USA, 2015, pp. 447-452. https://doi.org/10.1145/2684822.2697035.

[28] K. Chakraborty, S. Bhatia, S. Bhattacharyya, J. Platos, R. Bag, and A. E. Hassanien, "Sentiment Analysis of COVID-19 tweets by Deep Learning Classifiers-A study to show how popularity is affecting accuracy in social media," Appl. Soft Comput., vol. 97, p. 106754, 2020. https://doi.org/10.1016/j.asoc.2020.106754.

[29] A. Al Sallab, H. Hajj, G. Badaro, R. Baly, W. El Hajj, and K. B. Shaban, "Deep learning models for sentiment analysis in Arabic," in Proceedings of the Second Workshop on Arabic Natural Language Processing, 2015, pp. 9-17. https://doi.org/10.18653/v1/W15-3202

[30] A. Kumar, K. Srinivasan, W.-H. Cheng, and A. Y. Zomaya, "Hybrid context enriched deep learning model for fine-grained sentiment analysis in textual and visual semiotic modality social data," Inf. Process. Manag., vol. 57, no. 1, p. 102141, 2020. https://doi.org/10.1016/j.ipm.2019.102141. 
